\title{
The Polish Interventional Cardiology TAVI Survey (PICTS): adoption and practice of transcatheter aortic valve implantation in Poland
}

\author{
Radosław Parma ${ }^{1}$, Maciej Dąbrowski², Andrzej Ochała ${ }^{1}$, Adam Witkowski², Dariusz Dudek², \\ Zbigniew Siudak ${ }^{3}$, Jacek Legutko ${ }^{4}$ \\ ${ }^{1} 3^{\text {rd }}$ Department of Cardiology, Medical University of Silesia, Katowice, Poland \\ ${ }^{2}$ Department of Interventional Cardiology and Angiology, Institute of Cardiology, Warsaw, Poland \\ ${ }^{3}$ Department of Interventional Cardiology, Jagiellonian University Medical College, Kraków, Poland \\ ${ }^{4} 2^{\text {nd }}$ Department of Cardiology, Jagiellonian University Medical College, Kraków, Poland
}

Adv Interv Cardiol 2017; 13, 1 (47): 10-17

DOI: https://doi.org/10.5114/aic.2017.66181

\begin{abstract}
A bstract
Introduction: Few studies have assessed the development of transcatheter aortic valve implantation (TAVI) in Poland since its introduction in 2008, and data on current TAVI activity or practice are missing.

Aim: To assess the dynamics of TAVI adoption in Poland and to detect differences among Polish centres in TAVI practice and decision-making.

Material and methods: The Polish Interventional Cardiology TAVI Survey (PICTS) was approved by the Polish Association of Cardiovascular Interventions and presented to all 21 national TAVI centres. Between 2008 and 2015 the cumulative number of TAVI performed in Poland was 2189. The annual number of TAVI rose from 8 in 2008 to 670 in 2015 (0.21 to 17.4 implants per million inhabitants, respectively).

Results: The median TAVI experience per centre was 80 procedures $(95 \% \mathrm{Cl}: 38.1-154.6)$. In 2015 the TAVI penetration rate reached $5.12 \%$ of the estimated eligible Polish population. Inoperable and high-risk patients are treated with TAVI in all centres, with $52 \%$ of Heart Teams also qualifying medium-risk patients. The rate of transfemoral implantations increased to $83.2 \%$ of all procedures in 2015 , while transapical implantations decreased to $12 \%$. The frequency of subclavian, direct aortic or transcarotid routes in 2015 was below 3\% each.

Conclusions: The PICTS survey observed a positive but slow rate of adoption of TAVI in Poland. When compared to Western European countries, our findings highlight a significant treatment gap in high or prohibitive surgical risk patients with severe aortic stenosis. Remarkable variations in TAVI practices among Polish TAVI centres warrant publication of joint national guidelines and recommendations.
\end{abstract}

Key words: heart failure, transcatheter aortic valve implantation, transcatheter aortic valve replacement, aortic valve stenosis, Heart Team, aortic valve regurgitation.

\section{Introduction}

Since the first-in-man procedure performed in 2002, transcatheter aortic valve implantation (TAVI) has become the treatment of choice for inoperable patients and an alternative for high-risk patients with severe, symptomatic aortic stenosis [1, 2]. However, variations in regulatory, economic and social circumstances, as well as disease prevalence, influenced the disparity in TAVI adoption and practice in European countries [3]. So far, few studies have assessed TAVI development in Poland, and there is a paucity of data on TAVI activity and practice in the countries of Central and Eastern Europe [3-5]. The Association of Cardiovascular Intervention of the Polish Cardiac Society (ACVI) sought to address this gap by assessing the progress and current status of TAVI in Poland through a national web-based survey.

\section{Aim}

Specific aims of the study were to analyse dynamics of TAVI adoption in Poland since its introduction in 2008

\section{Corresponding author:}

Jacek Legutko MD, PhD, 2 ${ }^{\text {nd }}$ Department of Cardiology, Jagiellonian University Medical College, 17 Kopernika St, 31-501 Krakow, Poland, phone: +48 1242471 81, e-mail: jacek.legutko@uj.edu.pl

Received: 23.11.2016, accepted: 30.12.2016. 
and to detect differences among Polish centres in practice and decision-making in percutaneous aortic valve therapy.

\section{Material and methods \\ Data sourcing}

The survey was designed to investigate TAVI activity and practices in Polish interventional cardiology centres. It consisted of 41 single and multiple-choice questions focused on the following topics: 1 . characteristics of centres involved in the TAVI programme in Poland; 2. the annual number of TAVI implants from 2008 to 2015; 3. practice of pre-, intra- and post-procedural patient management. It was obligatory to answer all questions with the possibility to comment on any of them. After approval of the questionnaire by the ACVI in January 2016, the survey was published online at the ACVI official website, and formal invitations were sent to all invasive cardiology centres involved in the TAVI programme in Poland to participate. Responses were collected electronically by the end of February 2016.

\section{Transcatheter aortic valve implantation penetration}

We determined TAVI penetration rate as a measure of TAVI use relative to its use in patients with symptomatic severe aortic stenosis at high or excessive surgical risk that could potentially be treated with TAVI [6]. Following the already published methodology, among elderly inhabitants aged $\geq 75$ years with severe aortic stenosis (3.4\%), $75.6 \%$ were estimated to be symptomatic, $40.5 \%$ were deemed to be inoperable due to excessive surgical risk, and $5.2 \%$ were determined to be at high operative risk. $40.3 \%$ of inoperable patients and $80 \%$ of the high-risk patients were deemed to be potential TAVI candidates. Polish Central Statistical Office reports were used to esti-

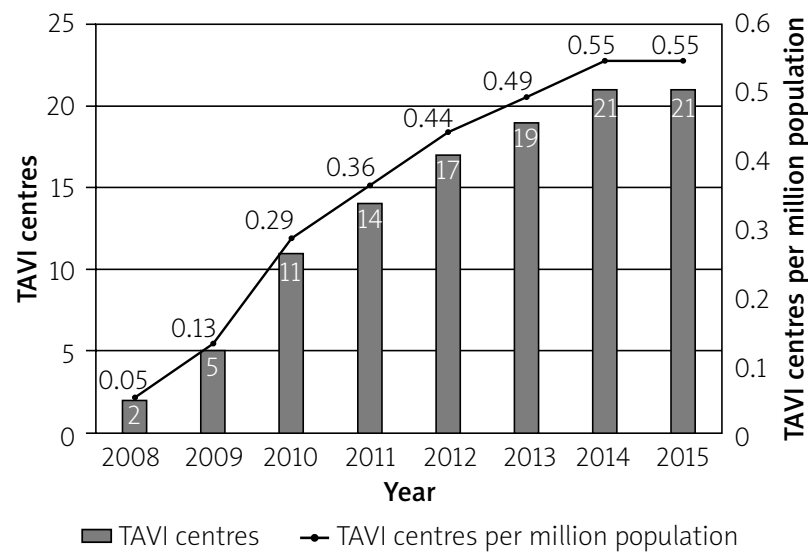

Figure 1. A cumulative number of transcatheter aortic valve implantation (TAVI) centres and number of TAVI centres per million population in Poland from 2008 to 2015 mate the size of the elderly population aged $\geq 75$ years for TAVI penetration analysis as well as to calculate the annual number of TAVI implants per million population and the number of TAVI centres per million population in Poland.

The results are presented in the paper as descriptive statistics.

\section{Results}

\section{Description of centres}

The TAVI programme was introduced in Poland in December 2008 and expanded across 21 centres by the end of 2015 (Figure 1). The number of TAVI centres per million population increased from 0.05 in 2008 to 0.55 in 2015. All of them fully participated in the survey: 15 (71\%) university centres, 5 (24\%) community hospitals and 1 (5\%) private hospital. Following recommendations of the European Society of Cardiology, 21 multidisciplinary Heart Teams involving 46 certified interventional cardiologists were established in all TAVI centres [7]. The distribution of TAVI experience across centres varied widely. The median cumulative number of TAVI implants per centre was 80 (95\% Cl: 38.1-154.6). From 2008 to 2015, 4 centres performed more than 200 (19\%) procedures, 4 centres performed between 100 and 200 procedures (19\%), while 13 centres performed less than 100 procedures (62\%; Figure 2). The median number of TAVI procedures per centre per annum increased from 4 in 2008 to 24 (95\% Cl: 13.7-41.4) in 2015. In the last year of survey, 5 centres performed $50-100$ procedures (24\%), 7 centres $20-49$ procedures (33\%) and 9 centres fewer than 20 procedures (43\%).

\section{Number of transcatheter aortic valve} implantation procedures

Between December 2008 and December 2015 the cumulative number of TAVI procedures performed in Poland

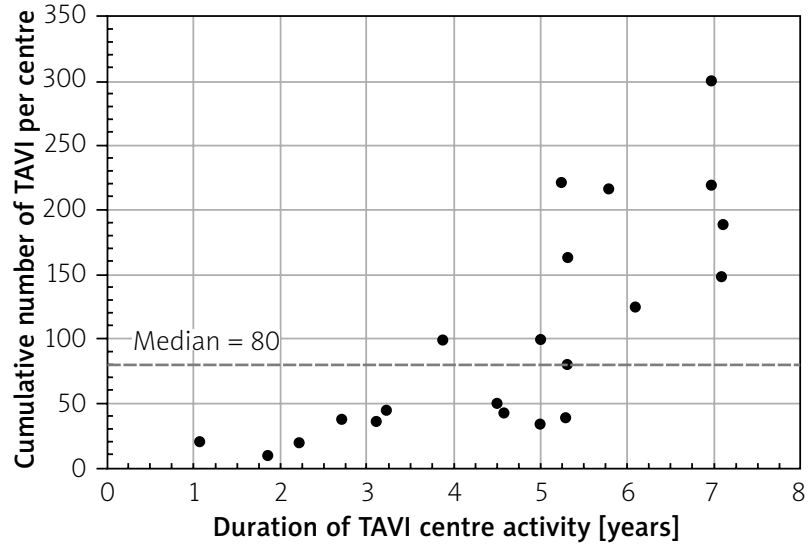

Figure 2. The TAVI activity in 21 Polish centres: Cumulative number of TAVI procedures per centre during years of activity (black markers represent single centres, median $=80$ procedures, grey line) 


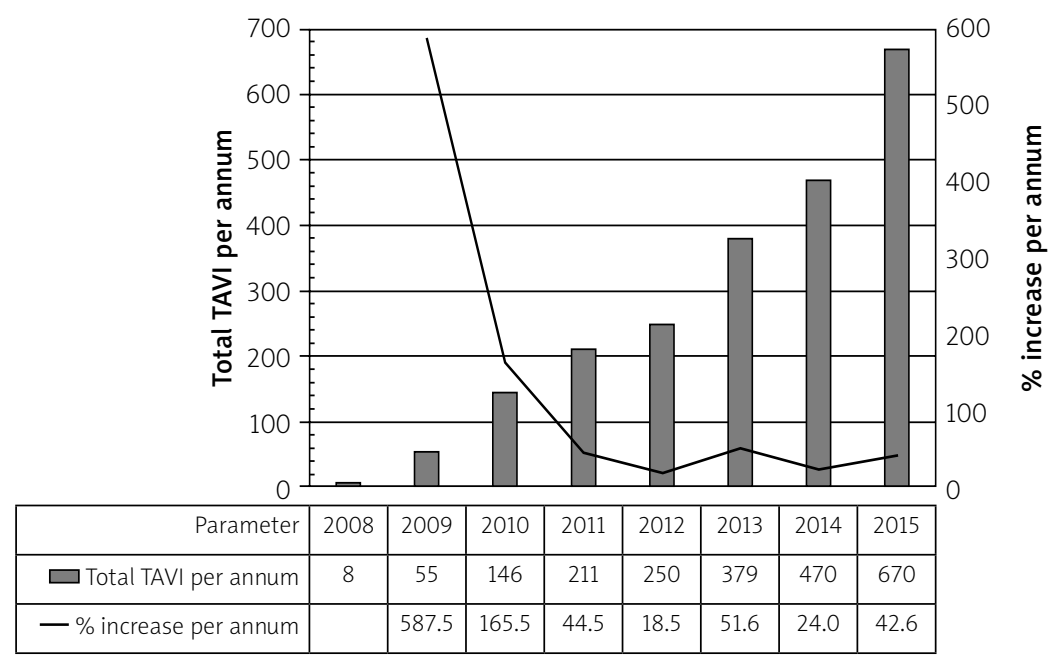

Figure 3. The TAVI procedures in Poland per annum and percentage of their annual increase

was 2189. The annual number of implantations increased from 8 in 2008 to 670 in 2015. The annual procedural volume growth rate was positive during all years, with a $42.6 \%$ increase in 2015 (Figure 3). The annual number of TAVI procedures per million inhabitants rose from 0.21 in 2008 to 17.4 in 2015. The annual number of TAVI implants per million inhabitants aged $\geq 75$ years increased from 3.2 in 2008 to 247.6 in 2015 (Figures 4 A and B).

\section{Transcatheter aortic valve implantation} penetration in Poland

Depending on Polish Central Statistical Office reports we estimated the number of potential TAVI candidates in the years from 2008 to 2015. Based on the number of TAVI recipients per annum, we calculated TAVI penetration rates presented in Table I. Consequently, out of 13076 TAVI eligible patients in 2015, 670 were treated with TAVI, leading to a $5.12 \%$ penetration rate in 2015 .

A

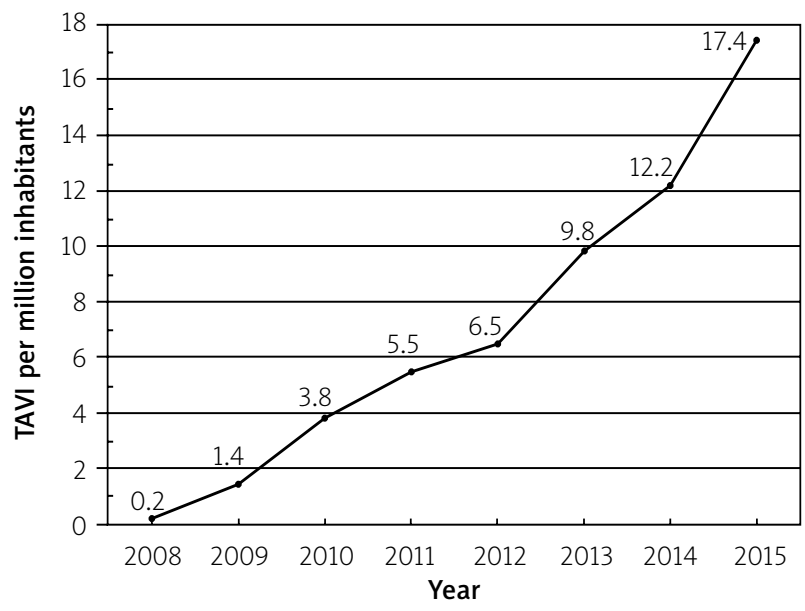

Transcatheter aortic valve implantation pre-procedural management

Risk

All Polish centres perform TAVI in inoperable and highrisk patients (Society of Thoracic Surgeons - STS score $>8$ ). Medium risk patients (STS 4-8) are qualified for TAVI in 10 centres $(52 \%)$, while low-risk patients (STS < 4) are offered transcatheter treatment in 1 centre (5\%).

\section{Imaging}

Multislice computed tomography (MSCT) is the imaging modality performed in all Polish centres to assess aortic valve anatomy before TAVI. In addition, transoesophageal echocardiography is concomitantly used in the majority of hospitals (66\%). Assessment of the vascular access route for TAVI is based on MSCT in $95 \%$ of centres, with angiography alone being used in one centre only $(5 \%)$.

\section{B}

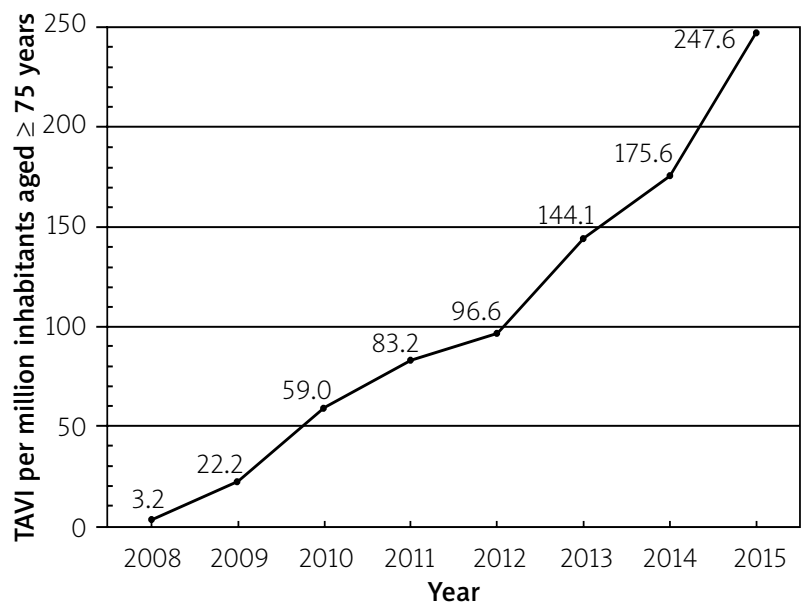

Figure 4. The TAVI procedures per annum in Poland from 2008 to 2015: A - TAVI implants per million inhabitants. B - TAVI implants per million inhabitants aged $\geq 75$ years 


\section{Coronary artery disease}

Coronary angiography is routinely performed in all centres as a part of pre-procedural planning. If patients are diagnosed with significant coronary artery disease, the strategy of preventive percutaneous coronary angioplasty of main vessels (PCI) is used by default in all centres. Nineteen percent of them may qualify such patients for simultaneous PCI during TAVI session, and only $14 \%$ of centres may decide on postponing $\mathrm{PCl}$ in such circumstance. After $\mathrm{PCl}$ with drug-eluting stent (DES), the recommended deferral of TAVI is 1 month in $71 \%$ of centres, 3 months in $24 \%$ and 6 months in $5 \%$ of centres.

In patients with coronary artery disease scheduled for TAVI with a femoral approach using surgical cutdown or closure devices, $95 \%$ of centres allow dual antiplatelet therapy (DAPT) to be maintained during TAVI. In patients qualified for transapical access, $60 \%$ of centres allow for DAPT, $35 \%$ recommend aspirin only and $5 \%$ withhold antiplatelet therapy on the day of the TAVI procedure.

\section{Transcatheter aortic valve implantation procedure}

\section{Prophylactic antibiotic therapy}

Routine use of periprocedural antibiotic therapy is advocated in $71 \%$ of centres. The remaining centres reserve antibiotic therapy for patients with additional indications.

\section{Anaesthesia}

Local anaesthesia for TAVI is used in $62 \%$ of centres. Since $2008,12.6 \%$ of patients have been treated with TAVI under local anaesthesia. In 2015 the rate of such procedures reached $20.6 \%$.

\section{Choice of access sites}

The transfemoral route is the default TAVI option in all Polish centres. Transapical access implantations have been performed in $95 \%$ of centres, followed by direct aortic (62\%) and subclavian routes (48\%). Only 3 centres have performed transcarotid TAVI in the last 2 years of the survey (14\%). The rate of transfemoral implantations increased to $83.4 \%$ of all procedures in 2015 , while the use of transapical access decreased to $11.8 \%$. The frequency of the subclavian, direct aortic or transcarotid route for TAVI in 2015 was below 3\% each (Figure 5).

\section{Use of closure devices}

Percutaneous closure devices are used for the transfemoral TAVI approach in $43 \%$ of centres. Perclose ProGlide Suture-Mediated Closure Systems (Abbott Vascular, Redwood City, CA, USA) is used by $29 \%$ of centres and a single Prostar XL Percutaneous Vascular Surgical Systems (Abbott Vascular, Redwood City, CA, USA) in 33\% of centres. Since the beginning of the TAVI programme

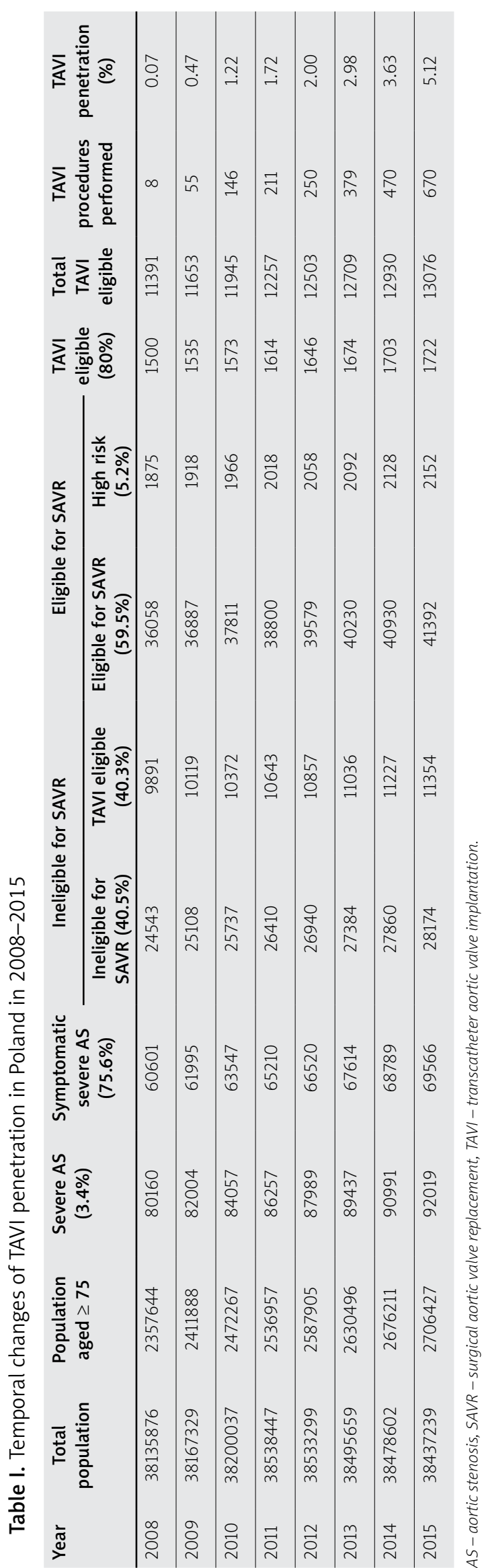




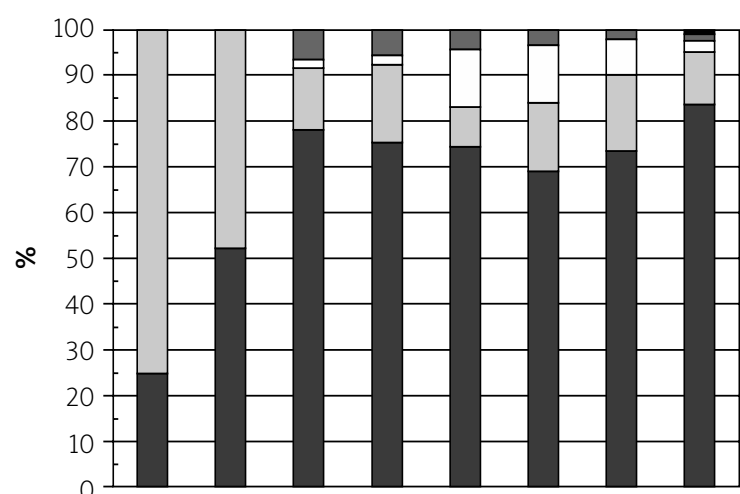

\begin{tabular}{|r|c|c|c|c|c|c|c|c|}
\hline Parameter & 2008 & 2009 & 2010 & 2011 & 2012 & 2013 & 2014 & 2015 \\
\hline - Transcarotid & 0.0 & 0.0 & 0.0 & 0.0 & 0.0 & 0.0 & 0.2 & 0.9 \\
\hline$\square$ Subclavian & 0.0 & 0.0 & 6.8 & 5.7 & 4.8 & 3.4 & 1.9 & 1.6 \\
\hline$\square$ Direct aortic & 0.0 & 0.0 & 1.4 & 1.9 & 12.0 & 12.4 & 7.7 & 2.2 \\
\hline$\square$ Transapical & 75.0 & 47.3 & 13.7 & 17.1 & 8.8 & 15.0 & 16.4 & 11.8 \\
\hline$\square$ Transfemoral & 25.0 & 52.7 & 78.1 & 75.4 & 74.4 & 69.1 & 73.8 & 83.4 \\
\hline
\end{tabular}

Figure 5. Temporal changes of access site use during TAVI in Poland per annum

in Poland percutaneous closure devices have been used in $31.6 \%$ of patients (Perclose Proglide $3.8 \%$, Prostar XL $27.8 \%)$. In 2015, closure devices were used in $28.1 \%$ of patients (Perclose Proglide 10.4\%, Prostar XL 17.7\%).

\section{Aortic balloon valvuloplasty before transcatheter aortic valve implantation}

Sixty-seven percent of centres consider TAVI without the use of aortic balloon valvuloplasty. As a result, $31 \%$ of patients were treated with direct TAVI in 2015.

\section{Transcatheter aortic valve implantation} post-procedural management

\section{Right ventricular electrode removal after transcatheter aortic valve implantation}

In patients without evidence of new conduction system abnormalities, $48 \%$ of centres maintain the rightventricular electrode for 2 days after the TAVI procedure, $38 \%$ remove it after $24 \mathrm{~h}$ and $4 \%$ discard it in the hybrid room.

\section{Double antiplatelet therapy or anticoagulation after transcatheter aortic valve implantation}

The antithrombotic regimens prescribed after TAVI include DAPT in $81 \%$ and single antiplatelet therapy in $19 \%$ of centres. No centre is currently mandating oral anticoagulation therapy for TAVI indication only. In all patients with standard indications for anticoagulation, a single antiplatelet agent is prescribed in combination. Antiplatelet therapy is recommended for 3 months in $56 \%$ of responding centres, while a 6-month regime is supported by $44 \%$ of them.

\section{Discussion}

The Polish Interventional Cardiology TAVI Survey (PICTS) survey is the first report of TAVI adoption and practice in Poland since its introduction in 2008. The main findings are as follows: 1 . The number of TAVI centres per million population is 0.05 with substantial variation in the number of TAVI implants per centre across Poland: the median cumulative number of TAVI implants per centre was 80 (95\% Cl: 38.1-154.6); 2. The annual number of TAVI procedures per million population increased from 0.21 in 2008 to 17.4 in 2015; 3. TAVI remains greatly underutilised with an estimated weighted penetration rate of $5.12 \%$ in 2015 ; 4 . A vast majority of patients are treated with TAVI using the transfemoral route, with the rate of $83.4 \%$ in 2015; 5 . A broad divergence of procedural TAVI practices exists between national centres in Poland.

Organisation of Polish centres built around TAVI therapy adheres to current guidelines and recommendations with Heart Teams, interventional cardiology and cardiac surgery departments established in all of them [7, 8]. Although their number reached an average of Western European nations such as France, the United Kingdom and Denmark, the experience of Polish centres with median 24 TAVI performed in 2015 is below the European average of 41 procedures in 2011 [3]. We observed substantial variation of TAVI implantations per centre in Poland, with $19 \%$ of centres performing $50-100$ procedures and $33 \%$ of centres performing fewer than 20 implants in 2015. Eleven centres performed fewer than 50 procedures in total despite their long activity (Figure 2). The causes of such restraints were beyond the scope of the study. As all centres reported long patient waiting lists, TAVI quota and reimbursement limits may be the likely causes of the slow progress rate.

Despite a positive growth of procedural volume from 2008 to 2015, we found that the latest annual number of 17.1 TAVI procedures per million population in Poland is 10 times lower than in Germany, 3 times lower than in Sweden, twice as low as in Ireland and half of the Western European average reported in 2011 [9-11]. Similarly, the estimated TAVI penetration rate of $5.12 \%$ in Poland in 2015 is lower than in any Western European country except for Portugal in 2011 [3]. According to our estimates, the number of TAVI eligible patients in Poland was 13076 in 2015. The published estimated number of new TAVI candidates each year in Poland, under current indications, was 1220 [6]. In contrast, the number of TAVI procedures performed in Poland in 2015 was 670. Based on preliminary reports, the European Association of Percutaneous Interventions (EAPCI) introduced the Valve for Life initiative in Poland in 2015 "to enhance awareness concerning inequality of patient access to the lifesaving indications of TAVI therapy [...] with the goal of increasing the treatment of severe valvular heart disease by $20 \%$ by 2020" [12]. Our survey observed a $42.6 \%$ increase of TAVI procedures in Poland in 2015 in comparison to 2014. 
Patient risk distribution in Polish centres resembles current European practice with universal acceptance of TAVI in patients with extreme and high risk, but also in selected medium risk patients [13]. The MSCT is the imaging technique of choice for aortic valve and vascular access route assessment, reflecting current guidelines and concordance with European standards [8, 14, 15]. Coronary revascularisation procedures $(\mathrm{PCl})$ in patients qualified for TAVI in Poland are performed before the procedure in all centres according to the actual European recommendations for revascularization [16]. The observed practice of $\mathrm{PCI}$ during TAVI used in $19 \%$ of centres or postponing $\mathrm{PCl}$ after TAVI present in $14 \%$ of centres may reflect the preference given to the treatment of aortic stenosis and incomplete evidence for management of coronary artery disease before TAVI [16, 17]. Variations in practice of maintaining dual antiplatelet therapy before TAVI using the transapical approach may mirror difficult decision-making in high-risk patients.

Contrary to recent reports of the German AQUA Registry of transapical use in $22.3 \%$ of patients qualified for TAVI, the number of Polish patients treated transapically decreased to $11.8 \%$ of all procedures in 2015 , while the transfemoral approach was predominant in Poland from 2009 onwards [10]. The factors influencing such changes may be technology advances in the design of femoral delivery systems and supportive long-term results of TAVI procedures in medium-risk patients using transfemoral access [18]. Infrequent use of alternative access sites (subclavian, direct aortic, transcarotid) may have been limited by the facilitated transfemoral approach and relatively low procedural volume in Polish centres.

The observed predominant use of surgical femoral artery cutdown over suture-based closure devices may be explained by assumed avoidance of vascular complications and bleeding as well as the decreasing cost of the procedure. Also, TAVI operators need thorough experience in using closure systems, which is possible to achieve with a high number of implantations. The reported preference of Prostar XL over Proglide use in Polish centres depends on local practice. Current conflicting reports on the safety of closure device use in TAVI suggest the need for further research in this field $[19,20]$.

The relatively large proportion of patients treated with direct valve implantation may represent the process of simplifying the TAVI procedure to increase its safety, but more evidence for such an approach is needed [21]. In fact, $33 \%$ of Polish centres perform aortic valvuloplasty during TAVI.

The DAPT after TAVI is currently recommended in most centres, while more evidence is needed on antithrombotic therapy [22]. There is a broad range of recommended duration of DAPT across national centres, with no consensus present also in a European survey in which $53 \%$ of centres supported a 3-month and 19\% a 6-month DAPT after valve implantation [13].
The survey has the following limitations, which should be considered when interpreting the results. Although the study was fully addressed by all Polish TAVI centres, the answers were provided mostly by interventional cardiologists and may not be fully representative of the whole multidisciplinary team community. The numbers of TAVI procedures reported were not validated with registries of the national health system administration in the years 2008-2013 due to the lack of such reports. The causes of the low TAVI penetration rate were not surveyed in the study and further research in this field is warranted. The estimates of TAVI use are likely to have included patients treated for off-label indications, such as patients at lower surgical risk, which may have affected the estimates of TAVI penetration. Direct comparisons to TAVI adoption practices in countries of Central and Eastern Europe were not described due to the absence of published reports.

\section{Conclusions}

The PICTS survey highlighted a positive but relatively slow rate of adoption of TAVI in Poland. When compared to Western European countries, our findings point to a large treatment gap in high or prohibitive surgical risk patients with severe aortic stenosis. In addition, remarkable regional variations in TAVI growth exist among Polish TAVI centres. Currently, the EAPCI Valve for Life campaign addresses the problem with multidirectional actions aiming to offer improved care to Polish patients. Finally, substantial discrepancies between practices of centres point to the need for publication of joint national guidelines and recommendations.

\section{Impact on daily practice}

Until now temporal changes of TAVI adoption and practice in countries of Central and Eastern Europe have remained largely unreported. The PICTS study highlights a slow rate of TAVI adoption in Poland in comparison to countries of Western Europe. We observed significant improvements of TAVI penetration after the introduction of the EAPCI Valve for Life campaign in Poland. The survey demonstrated wide variations in TAVI practice among Polish centres, calling for publication of joint TAVI practice guidelines.

\section{Acknowledgments}

The authors would like to thank the leaders of Polish TAVI Heart Teams for their help in providing datasets used in the PICTS study and their support given to the Valve-for-Life programme:

- Sławomir Dobrzycki, Paweł Kralisz, Marek Frank, Tomasz Hirnle, Medical University of Białystok, Białystok;

- Paweł Buszman, Marcin Dębiński, Wojciech Domaradzki, Marek Cisowski, American Heart of Poland, Bielsko-Biała; 
- Wojciech Pawliszak, Dr Antoni Jurasz Memorial University Hospital, Bydgoszcz;

- Robert Romanek, Maurycy Missima, $10^{\text {th }}$ Military Research Hospital, Bydgoszcz;

- Dariusz Ciećwierz, Dariusz Jagielak, Medical University of Gdansk, Gdańsk;

- Andrzej Ochała, Wojciech Wojakowski, Grzegorz Smolka, Radosław Parma, Marek Deja, Medical University of Silesia, Katowice;

- Dariusz Dudek, Jarosław Trębacz, Paweł Kleczyński, Robert Sobczyński, Bogusław Kapelak, Jagiellonian University Medical College, Kraków;

- Jarosław Wójcik, Andrzej Madejczyk, Janusz Stążka, Medical University of Lublin, Lublin;

- Michat Kidawa, Bogdan Jegier, Andrzej Walczak, Michał Krejca, Medical University of Lodz, Łódź;

- Jerzy Sacha, Jacek Kaperczak, Regional Medical Center, Opole;

- Maciej Lesiak, Marek Grygier, Marcin Misterski, Marek Jemielity, Poznan University of Medical Sciences, Poznań;

- Piotr Olszówka, Kazimierz Widenka, $2^{\text {nd }}$ District Hospital, Rzeszów;

- Jarosław Gorący, Maciej Lewandowski, Mirosław Brykczyński, Pomeranian Medical University, Szczecin;

- Wojciech Wąsek, Grzegorz Suwalski, Military Institute of Medicine, Warsaw;

- Robert Gil, Tomasz Pawłowski, Piotr Suwalski, Central Clinical Hospital of the Ministry of the Interior and Administration, Warsaw;

- Janusz Kochman, Zenon Huczek, Radosław Wilimski, Medical University of Warsaw, Warsaw;

- Adam Witkowski, Zbigniew Chmielak, Maciej Dąbrowski, Krzysztof Kuśmierski; Institute of Cardiology, Warsaw;

- Marcin Protasiewicz, Marek Jasiński, Wrocław Medical University, Wrocław;

- Krzysztof Reczuch, Artur Telichowski, Jacek Skiba, Clinical Military Hospital, Wrocław;

- Krzysztof Wilczek, Piotr Chodór, Marian Zembala, Medical University of Silesia, Zabrze;

- Andrzej Kleinrok, Tomasz Smyk, Łukasz Tułecki, Pope John Paul II Hospital, Zamość.

\section{Footnotes}

RP and MD contributed equally to this study. Study concept, design and supervision: all authors. Conduct of the study: RP, MD. Analysis and interpretation of data: RP, MD. Drafting and revision of the manuscript: all authors. Approval of the final version of the manuscript: all authors.

\section{Conflict of interest}

The authors declare no conflict of interest.

\section{References}

1. Cribier A, Eltchaninoff H, Bash A, et al. Percutaneous transcatheter implantation of an aortic valve prosthesis for calcific aortic stenosis. First Human Case Description 2002; 106: 3006-8.

2. Siontis GCM, Praz F, Pilgrim T, et al. Transcatheter aortic valve implantation vs. surgical aortic valve replacement for treatment of severe aortic stenosis: a meta-analysis of randomized trials. Eur Heart J 2016; 37: 3503-12.

3. Mylotte D, Osnabrugge RL, Windecker S, et al. Transcatheter aortic valve replacement in Europe: adoption trends and factors influencing device utilization. J Am Coll Cardiol 2013; 62: 210-9.

4. Osnabrugge RL, Kappetein AP, Reynolds MR, et al. Cost-effectiveness of transcatheter valvular interventions: economic challenges. Eurolntervention 2013; 9 Suppl: S48-54.

5. Di Mario C, Eltchaninoff H, Moat NE, et al. The 2011-12 pilot European Sentinel Registry of Transcatheter Aortic Valve Implantation: in-hospital results in 4,571 patients. Eurolntervention 2013; 8: 1362-71.

6. Osnabrugge RL, Mylotte D, Head SJ, et al. Aortic stenosis in the elderly: disease prevalence and number of candidates for transcatheter aortic valve replacement: a meta-analysis and modeling study. J Am Coll Cardiol 2013; 62: 1002-12.

7. Vahanian A, Alfieri O, Al-Attar N, et al. Transcatheter valve implantation for patients with aortic stenosis: a position statement from the European Association of Cardio-Thoracic Surgery (EACTS) and the European Society of Cardiology (ESC), in collaboration with the European Association of Percutaneous Cardiovascular Interventions (EAPCI). Eur Heart J 2008; 29: 1463-70.

8. Tommaso CL, Bolman RM 3rd, Feldman T, et al. Multisociety (AATS, ACCF, SCAI, and STS) expert consensus statement: operator and institutional requirements for transcatheter valve repair and replacement, part 1: transcatheter aortic valve replacement. J Am Coll Cardiol 2012; 59: 2028-42.

9. Mylotte D, Piazza N, Serruys PW. TAVI adoption in Germany: onwards and upwards. Eurolntervention 2016; 11: 968-70.

10. Eggebrecht $\mathrm{H}$, Mehta RH. Transcatheter aortic valve implantation (TAVI) in Germany 2008-2014: on its way to standard therapy for aortic valve stenosis in the elderly? Eurolntervention 2016; 11: 1029-33.

11. Rück A. SWEDEHEART Annual Report 2015: Percutaneous Valve Registry. In: Tillberg M (ed.). SWEDEHEART Annual Report 2015: Tomas Jernberg 2016; 298.

12. Windecker S, Haude M, Baumbach A. Introducing a new EAPCI programme: the valve for life initiative. Eurolntervention 2016; 11: 977-9.

13. Petronio AS, Capranzano P, Barbato E, et al. Current status of transcatheter valve therapy in Europe: results from an EAPCI survey. Eurolntervention 2016; 12: 890-5.

14. Achenbach S, Delgado V, Hausleiter J, et al. SCCT expert consensus document on computed tomography imaging before transcatheter aortic valve implantation (TAVI)/transcatheter aortic valve replacement (TAVR). J Cardiovasc Comput Tomogr 2012; 6: 366-80.

15. Dill KE, George E, Abbara S, et al. ACR appropriateness criteria imaging for transcatheter aortic valve replacement. J Am Coll Radiol 2013; 10: 957-65.

16. Abramowitz Y, Banai S, Katz G, et al. Comparison of early and late outcomes of TAVI alone compared to TAVI plus PCI in aortic stenosis patients with and without coronary artery disease. Catheter Cardiovasc Interv 2014; 83: 649-54. 
17. Gasparetto V, Fraccaro C, Tarantini G, et al. Safety and effectiveness of a selective strategy for coronary artery revascularization before transcatheter aortic valve implantation. Catheter Cardiovasc Interv 2013; 81: 376-83.

18. Leon MB, Smith CR, Mack MJ, et al. Transcatheter or surgical aortic-valve replacement in intermediate-risk patients. N Engl J Med 2016; 374: 1609-20.

19. Barbanti M, Capranzano P, Ohno Y, et al. Comparison of suture-based vascular closure devices in transfemoral transcatheter aortic valve implantation. Eurolntervention 2015; 11: 690-7.

20. Barbash IM, Barbanti M, Webb J, et al. Comparison of vascular closure devices for access site closure after transfemoral aortic valve implantation. Eur Heart J 2015; 36: 3370-9.

21. Bijuklic K, Haselbach T, Witt J, et al. Increased risk of cerebral embolization after implantation of a balloon-expandable aortic valve without prior balloon valvuloplasty. JACC Cardiovasc Interv 2015; 8: 1608-13.

22. Gargiulo G, Collet JP, Valgimigli M. Antithrombotic therapy in TAVI patients: changing concepts. Eurolntervention 2015; 11: W92-5. 